\title{
On the calculation of inner products of Schur functions
}

\author{
J.A. Castilho Alcarás* \\ Instituto de Física Teórica, Universidade Estadual Paulista, UNESP, 01140-070, São Paulo, Brazil
}

\author{
V.K.B. Kota ${ }^{\dagger}$ \\ Physical Research Laboratory, Ahmedabad 380 009, India
}

(Received on 30 September, 2009)

\begin{abstract}
Two methods for calculating inner products of Schur functions in terms of outer products and plethysms are given and they are easy to implement on a machine. One of these is derived from a recent analysis of the $S O(8)$ proton-neutron pairing model of atomic nuclei. The two methods allow for generation of inner products for the Schur functions of degree up to 20 and even beyond.
\end{abstract}

Keywords: Group theory, Group Representation theory, Symmetric group.

\section{INTRODUCTION}

Quantum many-particle systems in general carry several degrees of freedom. For example atomic nuclei carry orbital, spin and isospin degrees of freedom. In group theoretical models of these systems (say with $n$ particles), the wavefunctions that correspond to these different degrees of freedom transform according to definite irreducible representations (irreps) of both the spectrum generating group $G$ and the permutation group $S_{n}$ of $n$ objects. These irreps are then coupled such that the total wave functions are completely antisymmetric (if we have fermion systems) or symmetric (if we have boson systems) under permutation of all particles. For recent applications of group theoretical models to fermion and boson systems, see [1,2]. Therefore, one needs to know how to reduce the Kronecker product of two $S_{n}$ irreps into $S_{n}$ irreps and this gives the Clebsch-Gordon series for the symmetric groups [3]. In group theory this product corresponds to inner product of Schur functions $[4,5]$. The $S_{n}$ irreps are labeled by the partitions $(\lambda)=\left(\lambda_{1}, \lambda_{2}, \ldots, \lambda_{n}\right)$ of the integer $n$. Note that $\lambda_{1} \geq \lambda_{2} \geq \ldots \geq \lambda_{n} \geq 0$ and $\sum_{i=1}^{n} \lambda_{i}=n$. Our purpose in this paper is to give methods for evaluating the Kronecker products of $S_{n}$ irreps with $n$ up to 20 and beyond. Before going further it is useful to mention that the spectrum generating groups $G$ of interest are general linear groups $G L(N)$, unitary groups $U(N)$ and so on $[1,2,6]$.

A powerful tool in the study of the representations of unitary groups and their subgroups including the $S_{n}$ groups, is Schur functions $[5,7,8]$ and they are characters of the unitary irreps of unitary groups (also the characters of finite dimensional irreps of general linear groups) $[6,7]$. The Schur functions are symmetric homogeneous polynomials in $p$ indeterminates (variables) $\mathbf{x}=\left(x_{1}, x_{2}, \ldots, x_{p}\right)$ and more importantly they involve a partition $(\lambda)$ of an integer $r$ having $k$ parts, i.e. $(\lambda)=\left(\lambda_{1}, \lambda_{2}, \ldots, \lambda_{k}\right)$ such that $\lambda_{k}>0, p \geq k$ and $r=\sum_{i=1}^{k} \lambda_{i}$. Now a comprehensible definition of the Schur functions $\{\lambda\}_{\mathrm{X}}$ of degree $r$, in terms of determinants is $[6,7]$

$$
\{\lambda\}_{\mathbf{x}}=\frac{|M(\lambda)|}{|M(\lambda=0)|}
$$

where $M(\lambda)$ is a $p \times p$ matrix with elements $M_{i j}(\lambda)=$ $\left(x_{i}\right)^{\lambda_{j}+p-j}$. Littlewood established many properties of Schur functions and they allow us to deal with these functions without using the explicit expression given by Eq. (1) in terms of the variables $\mathbf{x}$. Because of this reason, Schur functions $\{\lambda\}_{\mathbf{x}}$ are simply denoted by $\{\lambda\}$.

Given two Schur functions $\{\lambda\}$ and $\{\mu\}$, they can be 'multiplied' in three different ways giving: (i) outer product denoted by $\{\lambda\}\{\mu\}=\sum_{v} \Gamma_{\lambda \mu v}\{v\}$; (ii) inner product denoted by $\{\lambda\} \times\{\mu\}=\sum_{v} g_{\lambda \mu v}\{v\}$; (iii) plethysm denoted by $\{\lambda\} \otimes\{\mu\}=\sum_{v} \Phi_{\lambda \mu \nu}\{v\}$. The outer product gives the reduction of the Kronecker product (sometimes called tensor product) of $U(n)$ irreps $\{\lambda\}$ and $\{\mu\}$ into $U(n)$ irreps $\{v\}$ and $\Gamma_{\lambda \mu v}$ is the multiplicity (or number of occurrences) of the irrep $\{v\}$ in the product. Note that here the irreps $\{\lambda\}$ and $\{v\}$ correspond to partitions $(\lambda)$ and $(\mu)$ for two different integers $n_{1}$ and $n_{2}$ and $\{v\}$ correspond to the partition $(v)$ for the integer $n_{1}+n_{2}$. Similarly the inner product gives the reduction of the Kronecker product of $S_{n}$ irreps labeled by partitions $(\lambda)$ and $(\mu)$ into $S_{n}$ irreps $(v)$ and $g_{\lambda \mu v}$ is the multiplicity of the irrep $(v)$. The third product is called plethysm or symmetrized power and it is explained in [5-7, 9-11]. For example say the states of a single particle correspond to the irrep $\{\lambda\}$ of $U(n)$. Then with $m$ identical particles occupying these states, the $m$ particle states with permutation symmetry $(\mu)$ of $S_{m}$ transform as the irreps $\{v\}$ of $U(n)$ and the $\{v\}$ are given by the plethysm $\{\lambda\} \otimes\{\mu\}$. It should be stressed that, although the subject of Schur function multiplications is old, there is recent interest in developing algorithms for these $[6,11-14]$ and this is mainly due to the developments in group theoretical models for atomic nuclei.

The coefficients $\Gamma_{\lambda \mu v}$ of outer products can be obtained by the well known Littlewood Richardson rules $[8,15]$ and it is easy develop a computer code using these rules. In addition, recently computer codes are also developed for evaluating plethysms [11-13] and one of the authors [16] has created a bank of plethysms. On the other hand inner products are more complicated. By definition the $g_{\lambda \mu v}$ in the inner product are given by the formula $[3,5]$

$$
g_{\lambda^{\prime} \lambda^{\prime \prime} \lambda}=\sum_{\beta} \frac{h(\beta)}{n !} \chi^{\left(\lambda^{\prime}\right)}(\beta) \chi^{\left(\lambda^{\prime \prime}\right)}(\beta) \chi^{(\lambda)}(\beta) .
$$

In Eq. (2) the sum is over the $S_{n}$ classes $\beta, h(\beta)$ are their order and $\chi^{(\lambda)}(\beta)$ their character in the irrep $(\lambda)$. However this formula is difficult to use in practice for larger integer

\footnotetext{
*Electronic address: jaca@ift.unesp.br

${ }^{\dagger}$ Electronic address: vkbkota@prl.res.in
} 
values $n$. The three operations, outer product, inner product and plethysm are connected among themselves by relations known in the literature and exploiting this, we will give in this paper two methods for the calculation of inner products for irreps for large $n$ values. One of the methods discussed ahead is developed following a recent study of the $S O(8)$ proton-neutron pairing model of atomic nuclei [1]. In [5] tables for inner products are given for $n \leq 9$ only and the methods presented in the present paper allow one to go up to $n=20$ and beyond. It should be mentioned that in some special cases, formulas for inner products are available in literature [3,17]. Also, computer codes in $[12,13]$ allow one to calculate inner products but it is not clear if these codes allow one to consider partitions of integers up-to 20 and beyond. Now we will give the preview.

Section II gives the method based on outer products for calculating inner products. In Section III described is the method that uses both plethysms and outer products. In Section IV we present some examples for $n$ up to 20. Finally Section $\mathrm{V}$ gives conclusions.

\section{INNER PRODUCTS OF SCHUR FUNCTIONS IN TERMS OF OUTER PRODUCTS}

Our first method (I) uses the following equation, obtained by Littlewood[4, 5], and it allows us to compute inner products of Schur functions using only outer products,

$$
(\{\mu\}\{\lambda\}) \times\{v\}=\sum_{\rho, \sigma} \Gamma_{\rho \sigma v}(\{\mu\} \times\{\rho\})(\{\lambda\} \times\{\sigma\}) .
$$

In Eq. (3), $\Gamma_{\rho \sigma v}$ is the multiplicity of $\{v\}$ in the outer product $\{\rho\}\{\sigma\}$ of $\{\rho\}$ by $\{\sigma\}$. To use this equation say for $\{\mu\} \times$ $\{\lambda\}$, one starts by singling out the last non-null part $\mu_{t}$ of $\mu$, and then write using Eq.(3),

$$
\begin{aligned}
& \left(\left\{\mu_{1}, \mu_{2}, \ldots, \mu_{t-1}\right\}\left\{\mu_{t}\right\}\right) \times\{\lambda\}= \\
& \sum_{\lambda^{\prime}, \lambda^{\prime \prime}} \Gamma_{\lambda^{\prime} \lambda^{\prime \prime} \lambda} \lambda\left(\left\{\mu_{1}, \mu_{2}, \ldots, \mu_{t-1}\right\} \times\left\{\lambda^{\prime}\right\}\right)\left(\left\{\mu_{t}\right\} \times\left\{\lambda^{\prime \prime}\right\}\right) \\
& =\sum_{\lambda^{\prime} \lambda^{\prime \prime}} \Gamma_{\lambda^{\prime} \lambda^{\prime \prime} \lambda}\left(\left\{\mu_{1}, \mu_{2}, \ldots, \mu_{t-1}\right\} \times\left\{\lambda^{\prime}\right\}\right)\left\{\lambda^{\prime \prime}\right\} .
\end{aligned}
$$

In the last step we used $\left\{\mu_{t}\right\} \times\left\{\lambda^{\prime \prime}\right\}=\left\{\lambda^{\prime \prime}\right\}$ since $\left\{\mu_{t}\right\}$ is symmetric. On the other hand,

$$
\left\{\mu_{1}, \mu_{2}, \ldots, \mu_{t-1}\right\}\left\{\mu_{t}\right\}=\{\mu\}+\sum_{\mu^{\prime} \neq \mu} \Gamma_{\left\{\mu_{1}, \mu_{2}, \ldots, \mu_{t-1}\right\}\left\{\mu_{t}\right\}\left\{\mu^{\prime}\right\}}\left\{\mu^{\prime}\right\}
$$

implying that

$$
\begin{aligned}
& \left(\left\{\mu_{1}, \mu_{2}, \ldots, \mu_{t-1}\right\}\left\{\mu_{t}\right\}\right) \times\{\lambda\}= \\
& \{\mu\} \times\{\lambda\}+\sum_{\mu^{\prime} \neq \mu} \Gamma_{\left\{\mu_{1}, \mu_{2}, \ldots, \mu_{t-1}\right\}\left\{\mu_{t}\right\}\left\{\mu^{\prime}\right\}}\left(\left\{\mu^{\prime}\right\} \times\{\lambda\}\right) .
\end{aligned}
$$

Now, equating Eq. (4) and Eq. (6) one obtains

$$
\begin{aligned}
& \{\mu\} \times\{\lambda\}=\sum_{\lambda^{\prime}, \lambda^{\prime \prime}} \Gamma_{\lambda^{\prime} \lambda^{\prime \prime} \lambda}\left(\left\{\lambda^{\prime}\right\} \times\left\{\mu_{1}, \mu_{2}, \ldots, \mu_{t-1}\right\}\right)\left\{\lambda^{\prime \prime}\right\}- \\
& \sum_{\mu^{\prime} \neq \mu} \Gamma_{\left\{\mu_{1}, \mu_{2}, \ldots, \mu_{t-1}\right\}\left\{\mu_{t}\right\}\left\{\mu^{\prime}\right\}}\left(\left\{\mu^{\prime}\right\} \times\{\lambda\}\right) .
\end{aligned}
$$

The inner products that appear in the first sum of Eq. (7) are from $S_{\left(n-\mu_{t}\right)}$ while those in the second sum are from
$S_{n}$. One can then establish a recursive procedure in which we compute all the $S_{n}$ inner products knowing those of $S_{n-1}, S_{n-2}, \ldots, S_{2}$. When computing inner products of a given $S_{n}$ irrep $\{\mu\}$ by all $\{\lambda\}$ one must be sure that all $S_{n}$ inner products appearing in the second sum of Eq. (7) have already been computed. This is achieved by ordering the irreps $\{\mu\}$ in decreasing order of their length and irreps of the same length being ordered in decreasing order of their parts read from left to right. The procedure then is:

1. Make a table of all outer products with final degree $n$ . This will give the coefficients $\Gamma$ and the irreps $\left\{\lambda^{\prime}\right\}$ and $\left\{\lambda^{\prime \prime}\right\}$ in the first sum in Eq. (7).

2. Make a table of all inner product for $S_{n-1}, S_{n-2}, \ldots, S_{2}$

3. Order all $S_{n}$ irreps in the order described above.

4. For a given $\{\lambda\}$, compute $\{\mu\} \times\{\lambda\}$ for all $\{\mu\}$ in the order given above using the formula

$$
\begin{aligned}
& \{\mu\} \times\{\lambda\}=\sum_{\lambda^{\prime}, \lambda^{\prime \prime}} \Gamma_{\lambda^{\prime} \lambda^{\prime \prime} \lambda}\left(\left\{\lambda^{\prime}\right\} \times\left\{\mu_{1}, \mu_{2}, \ldots, \mu_{t-1}\right\}\right)\left\{\lambda^{\prime \prime}\right\}- \\
& \sum_{\mu^{\prime} \prec \mu} \Gamma_{\left\{\mu_{1}, \mu_{2}, \ldots, \mu_{t-1}\right\}\left\{\mu_{t}\right\}\left\{\mu^{\prime}\right\}}\left(\left\{\mu^{\prime}\right\} \times\{\lambda\}\right) .
\end{aligned}
$$

In Eq. (8) $\prec$ means preceding following the order in step (3). Note that the procedure starts with the known result $\{\mu\} \times\{n\}=\{\mu\}$. Implementing the steps (1)-(4), a computer code has been developed; see ahead for examples. The calculation of inner products and construction of tables have been shortened by use of the well known properties of inner products [3],

$$
\begin{aligned}
& \{\mu\} \times\{\lambda\}=\{\lambda\} \times\{\mu\}, \\
& (\{\mu\} \times\{\lambda\})^{T}=\{\lambda\} \times\{\mu\}^{T}=\{\lambda\}^{T} \times\{\mu\}, \\
& \{\mu\}^{T} \times\{\lambda\}^{T}=\{\mu\} \times\{\lambda\}, \\
& \{n\} \times\{\lambda\}=\{\lambda\}, \quad\left\{1^{n}\right\} \times\{\lambda\}=\{\lambda\}^{T}
\end{aligned}
$$

where $\{f\}^{T}$ means transposed(or conjugate) of $\{f\}$.

\section{INNER PRODUCTS OF SCHUR FUNCTIONS IN TERMS OF PLETHYSMS}

Now we will turn to the second (II) method and this makes use of plethysms $[5,7,9,18]$. From the properties of plethysms one has [5],

$$
\left(\{1\}^{\prime}\{1\}^{\prime \prime}\right) \otimes\{\lambda\}=\sum_{\lambda^{\prime}, \lambda^{\prime \prime}} g_{\lambda^{\prime} \lambda^{\prime \prime} \lambda}\left\{\lambda^{\prime}\right\}^{\prime}\left\{\lambda^{\prime \prime}\right\}^{\prime \prime}
$$

where the symbol $\otimes$ means plethysm and the superscripts ' ', and 'II' over the Schur functions indicate that they are defined in different spaces (for example spin and orbital for atoms). If we can compute the LHS of Eq. (10) by other means, the comparison of results will give us the coefficients $g_{\lambda^{\prime} \lambda^{\prime \prime} \lambda}$ of the reduction of the inner product of $S_{n}$ irreps $\{\lambda\}^{\prime}$ and $\{\lambda\}^{\prime \prime}$. The result obtained from Eq. (10) can be dimensionally checked using (see Eq. (56) in [11] and also [14]),

$$
\sum_{\lambda^{\prime} \lambda^{\prime \prime}} g_{\lambda^{\prime} \lambda^{\prime \prime} \lambda} \operatorname{dim}\left\{\lambda^{\prime}\right\}_{n} \operatorname{dim}\left\{\lambda^{\prime \prime}\right\}_{n}=\operatorname{dim}\{\lambda\}_{n^{2}} .
$$


In Eq. (11) $\operatorname{dim}\{f\}_{r}$ is the dimension of the irrep $\{f\}$ of $U(r)$. Knowing the multiplicity coefficients $g$ 's for all the Schur functions of a given degree $n$, one constructs a table having in its columns these coefficients. The rows of such a table will give the reduction of the inner products of all Schur functions of degree $n$. We have recently used this trick in Ref. [1] for two-columns irreps $\{\lambda\}=\left\{2^{a} 1^{b}\right\}$ that appear in the so called $S O(8)$ model of atomic nuclei and this model describes pairing in $L S T$ coupling for nuclei; note that $T$ is isospin. The simplicity here is that it is possible to use the relation

$$
\left\{2^{a} 1^{b}\right\}=\left\{1^{a+b}\right\}\left\{1^{a}\right\}-\left\{1^{a+b+1}\right\}\left\{1^{a-1}\right\}, a>0
$$

and then Eq. (10) becomes

$$
\begin{aligned}
& \{1\}^{\prime}\{1\}^{\prime \prime} \otimes\left\{2^{a} 1^{b}\right\}= \\
& \left(\{1\}^{\prime}\{1\}^{\prime \prime}\right) \otimes\left(\left\{1^{a+b}\right\}\left\{1^{a}\right\}\right)-\left(\{1\}^{\prime}\{1\}^{\prime \prime}\right) \otimes\left(\left\{1^{a+b+1}\right\}\left\{1^{a-1}\right\}\right) \\
& =\left[\left(\{1\}^{\prime}\{1\}^{\prime \prime}\right) \otimes\left\{1^{a+b}\right\}\right]\left[\left(\{1\}^{\prime}\{1\}^{\prime \prime}\right) \otimes\left\{1^{a}\right\}\right] \\
& -\left[\left(\{1\}^{\prime}\{1\}^{\prime \prime}\right) \otimes\left\{1^{a+b+1}\right\}\right]\left[\left(\{1\}^{\prime}\{1\}^{\prime \prime}\right) \otimes\left\{1^{a-1}\right\}\right] .
\end{aligned}
$$

In Eq. (13) all plethysms have only antisymmetric Schur functions as right factors and for them one has the simple result

$$
\left(\{1\}^{\prime}\{1\}^{\prime \prime}\right) \otimes\left\{1^{p}\right\}=\sum_{f_{1}+f_{2}=p}\left\{2^{f_{2}}, 1^{f_{1}-f_{2}}\right\}^{\prime}\left\{f_{1}, f_{2}\right\}^{\prime \prime} .
$$

For example, Eq. (14) has been used in atomic physics (i.e. for fermion systems) in $L S$-coupling [5] and in nuclear physics while dealing with isospin in LST coupling [1]. Using Eq. (14) in Eq. (13) and performing the resulting outer products, the LHS of Eq. (10) is easily obtained for twocolumns irreps $\{\lambda\}$. Similar to Eqs. (12), (13) and (14), it is possible to obtain for a two rowed irrep $\left\{f_{1} f_{2}\right\}$, the plethysm $\{1\}^{\prime}\{1\}^{\prime \prime} \otimes\left\{f_{1} f_{2}\right\}$ by using the relation

$$
\left\{f_{1} f_{2}\right\}=\left\{f_{1}\right\}\left\{f_{2}\right\}-\left\{f_{1}+1\right\}\left\{f_{2}-1\right\}, f_{2}>0,
$$

and the basic result (used often for boson systems [5, 11]),

$$
g_{\lambda^{\prime} \lambda^{\prime \prime}\{m\}}=\delta_{\{\lambda\}^{\prime}\{\lambda\}^{\prime \prime}} .
$$

For general irreps $\{\lambda\}$ of degree $n$, it is possible to use the method based on Eq. (10) as it is possible to expand any Schur function $\{\lambda\}$ in terms of totally antisymmetric or symmetric Schur functions. This can be implemented in a recursive procedure by assuming that the results for all irreps of the integers 1 to $n-1$ are known and then obtain the inner products for the irreps of $n$. Alternatively one can use a procedure given in [11] to compute plethysms adapted for computing the LHS of Eq. (10) for all $\{\lambda\}$ of $S_{n}$ starting with

$$
\left(\{1\}^{\prime}\{1\}^{\prime \prime} \otimes\{n\}=\sum_{\lambda}\{\lambda\}^{\prime}\{\lambda\}^{\prime \prime} .\right.
$$

where use was made of Eq. (16) and the sum is over all $S_{n}$ irreps. Then the procedure is as follows.

In the first step (i), find all partitions of $n$ and order them in the order described after Eq. (7). Then in the second step (ii), for each partition $\{\lambda\}=\left\{\lambda_{1}, \lambda_{2}, \ldots, \lambda_{t}, 0, \ldots, 0\right\}$ perform the outer product $\left\{\lambda_{1}, \lambda_{2}, \ldots, \lambda_{t-1}\right\}\left\{\lambda_{t}\right\}$ and then use the equation

$$
\begin{aligned}
& \left(\{1\}^{\prime}\{1\}^{\prime \prime}\right) \otimes\{\lambda\}=\left[\left(\{1\}^{\prime}\{1\}^{\prime \prime}\right) \otimes\left\{\lambda_{1}, \lambda_{2}, \ldots, \lambda_{t-1}\right\}\right]\left[\left(\{1\}^{\prime}\{1\}^{\prime \prime}\right) \otimes\left\{\lambda_{t}\right\}\right] \\
& -\sum_{\left\{\lambda^{\prime}\right\} \prec\{\lambda\}} \Gamma_{\left\{\lambda_{1}, \lambda_{2}, \ldots, \lambda_{t-1}\right\}\left\{\lambda_{t}\right\} \rightarrow\left\{\lambda^{\prime}\right\}}\left[\left(\{1\}^{\prime}\{1\}^{\prime \prime}\right) \otimes\left\{\lambda^{\prime}\right\}\right]
\end{aligned}
$$

where the symbol $\prec$ means preceding, following the ordering in step (i). Now using Eq. (18) in Eq. (10), this procedure allows us to compute the inner products of all irreps of degree $n$, by induction, provided the inner products of irreps of degrees 1 to $n-1$ are known. In fact, using Eq. (18) we can compute all the inner products of $\{\lambda\}$ in the order of step (i), since all the $\left(\{1\}^{\prime}\{1\}^{\prime \prime}\right) \otimes\left\{\lambda^{\prime}\right\}$ in the RHS have been computed by the induction process.

\section{EXAMPLES FOR SCHUR FUNCTIONS OF DEGREE $n \leq 20$}

As a simple example (this can be worked out by hand calculations), first let us compute the inner product of all Schur functions of degree 4 using the inner products of Schur functions of degrees 1,2 and 3. Eq. (17) gives,

$$
\begin{aligned}
\left(\{1\}^{\prime}\{1\}^{\prime \prime}\right) \otimes\{4\} & =\{4\}^{\prime}\{4\}^{\prime \prime}+\{31\}^{\prime}\{31\}^{\prime \prime}+\left\{2^{2}\right\}^{\prime}\left\{2^{2}\right\}^{\prime \prime} \\
& +\left\{21^{2}\right\}^{\prime}\left\{21^{2}\right\}^{\prime \prime}+\left\{1^{4}\right\}^{\prime}\left\{1^{4}\right\}^{\prime \prime}
\end{aligned}
$$




$$
\begin{aligned}
& \left(\{1\}^{\prime}\{1\}^{\prime \prime}\right) \otimes\{31\}=\left[\left(\{1\}^{\prime}\{1\}^{\prime \prime}\right) \otimes\{3\}\right]\left[\left(\{1\}^{\prime}\{1\}^{\prime \prime}\right) \otimes\{1\}\right]-\left[\left(\{1\}^{\prime}\{1\}^{\prime \prime}\right) \otimes\{4\}\right] \\
& =\left[\{3\}^{\prime}\{3\}^{\prime \prime}+\{21\}^{\prime}\{21\}^{\prime \prime}+\left\{1^{3}\right\}^{\prime}\left\{1^{3}\right\}^{\prime \prime}\right]\left[\{1\}^{\prime}\{1\}^{\prime \prime}\right]-\left[\{4\}^{\prime}\{4\}^{\prime \prime}+\{31\}^{\prime}\{31\}^{\prime \prime}\right. \\
& \left.+\left\{2^{2}\right\}^{\prime}\left\{2^{2}\right\}^{\prime \prime}+\left\{21^{2}\right\}^{\prime}\left\{21^{2}\right\}^{\prime \prime}+\left\{1^{4}\right\}^{\prime}\left\{1^{4}\right\}^{\prime \prime}\right]=\{4\}^{\prime}\{31\}^{\prime \prime}+\{31\}^{\prime}\{31\}^{\prime \prime} \\
& +\{31\}^{\prime}\left\{2^{2}\right\}^{\prime \prime}+\{31\}^{\prime}\left\{21^{2}\right\}^{\prime \prime}+\left\{2^{2}\right\}^{\prime}\left\{21^{2}\right\}^{\prime \prime}+\left\{21^{2}\right\}^{\prime}\left\{21^{2}\right\}^{\prime \prime} \\
& +\left\{21^{2}\right\}^{\prime}\left\{1^{4}\right\}^{\prime \prime}+\text { xch terms }
\end{aligned}
$$

where 'xch terms' means exchange $\{\lambda\}^{\prime}$ by $\{\lambda\}^{\prime \prime}$ when they are different. Knowing $\left(\{1\}^{\prime}\{1\}^{\prime \prime}\right) \otimes\{31\}$ we use $\left\{2^{2}\right\}=$
$\{2\}\{2\}-\{4\}-\{31\}$ and obtain,

$$
\begin{aligned}
& \left(\{1\}^{\prime}\{1\}^{\prime \prime}\right) \otimes\left\{2^{2}\right\}=\left[\left(\{1\}^{\prime}\{1\}^{\prime \prime}\right) \otimes\{2\}\right]\left[\left(\{1\}^{\prime}\{1\}^{\prime \prime}\right) \otimes\{2\}\right]-\left[\left(\{1\}^{\prime}\{1\}^{\prime \prime}\right) \otimes\{4\}\right. \\
& \left.+\left(\{1\}^{\prime}\{1\}^{\prime \prime}\right) \otimes\{31\}\right]=\{4\}^{\prime}\left\{2^{2}\right\}^{\prime \prime}+\{31\}^{\prime}\{31\}^{\prime \prime}+\{31\}^{\prime}\left\{21^{2}\right\}^{\prime \prime}+\left\{2^{2}\right\}^{\prime}\left\{2^{2}\right\}^{\prime \prime} \\
& +\left\{2^{2}\right\}^{\prime}\left\{1^{4}\right\}^{\prime \prime}+\left\{21^{2}\right\}^{\prime}\left\{21^{2}\right\}^{\prime \prime}+\text { xch terms }
\end{aligned}
$$

From $\left\{21^{2}\right\}=\{2\}\left\{1^{2}\right\}-\{31\}$ one obtains,

$$
\begin{aligned}
& \left(\{1\}^{\prime}\{1\}^{\prime \prime}\right) \otimes\left\{21^{2}\right\}=\left[\left(\{1\}^{\prime}\{1\}^{\prime \prime}\right) \otimes\{2\}\right]\left[\left(\{1\}^{\prime}\{1\}^{\prime \prime}\right) \otimes\left\{1^{2}\right\}\right]-\left[\left(\{1\}^{\prime}\{1\}^{\prime \prime}\right) \otimes\{31\}\right] \\
& =\{4\}^{\prime}\left\{21^{2}\right\}^{\prime \prime}+\{31\}^{\prime}\{31\}^{\prime \prime}+\{31\}^{\prime}\left\{2^{2}\right\}^{\prime \prime}+\{31\}^{\prime}\left\{21^{2}\right\}^{\prime \prime}+\{31\}^{\prime}\left\{1^{4}\right\}^{\prime \prime}+\left\{2^{2}\right\}\left\{21^{2}\right\}^{\prime \prime} \\
& +\left\{21^{2}\right\}^{\prime}\left\{21^{2}\right\}^{\prime \prime}+\text { xch terms }
\end{aligned}
$$

Finally, $\left\{1^{4}\right\}=\left\{1^{3}\right\}\{1\}-\left\{21^{2}\right\}$ gives,

$$
\begin{aligned}
& \left(\{1\}^{\prime}\{1\}^{\prime \prime}\right) \otimes\left\{1^{4}\right\}=\left[\left(\{1\}^{\prime}\{1\}^{\prime \prime}\right) \otimes\left\{1^{3}\right\}\right]\left[\left(\{1\}^{\prime}\{1\}^{\prime \prime}\right) \otimes\{1\}\right]-\left[\left(\{1\}^{\prime}\{1\}^{\prime \prime}\right) \otimes\left\{21^{2}\right\}\right] \\
& =\{4\}^{\prime}\left\{1^{4}\right\}^{\prime \prime}+\{31\}^{\prime}\left\{21^{2}\right\}^{\prime \prime}+\left\{2^{2}\right\}^{\prime}\left\{2^{2}\right\}^{\prime \prime}+\text { xch terms }
\end{aligned}
$$

Now we order the RHS of Eqs. (19)-(23) in the order

$$
\begin{array}{r}
\{4\}^{\prime}\{4\}^{\prime \prime},\{4\}^{\prime}\{31\}^{\prime \prime},\{4\}^{\prime}\left\{2^{2}\right\}^{\prime \prime},\{4\}^{\prime}\left\{21^{2}\right\}^{\prime \prime},\{4\}^{\prime}\left\{1^{4}\right\}^{\prime \prime},\{31\}^{\prime}\{31\}^{\prime \prime},\{31\}^{\prime}\left\{2^{2}\right\}^{\prime \prime}, \\
\{31\}^{\prime}\left\{21^{2}\right\}^{\prime \prime},\{31\}^{\prime}\left\{1^{4}\right\}^{\prime \prime},\left\{2^{2}\right\}^{\prime}\left\{2^{2}\right\}^{\prime \prime},\left\{2^{2}\right\}^{\prime}\left\{21^{2}\right\}^{\prime \prime},\left\{2^{2}\right\}^{\prime}\left\{1^{4}\right\}^{\prime \prime},\left\{21^{2}\right\}^{\prime}\left\{21^{2}\right\}^{\prime \prime}, \\
\\
\left\{21^{2}\right\}^{\prime}\left\{1^{4}\right\}^{\prime \prime},\left\{1^{4}\right\}^{\prime}\left\{1^{4}\right\}^{\prime \prime}
\end{array}
$$

The coefficients of the resulting Eqs.(19)-(23) are used as entries in the columns of a table with columns labeled by $\{4\},\{31\},\left\{2^{2}\right\},\left\{21^{2}\right\},\left\{1^{4}\right\}$ and rows by the pairs $\{\lambda\}^{\prime}\{\lambda\}^{\prime \prime}$ given above. The rows in this table will give the coefficients of the expansion of $\{\lambda\}^{\prime} \times\{\lambda\}^{\prime \prime}$. Final results for $n=4$ are given in Table 1.
The methods I and II are implemented on a machine and inner products for $n \leq 20$ are obtained. As non-trivial examples, here below given are four examples for the inner products: (i) $\left\{5,4,2,1^{2}\right\} \times\left\{5,4,2,1^{2}\right\}$; (ii) $\{11,5\} \times\{6,4,3,2,1\}$; (iii) $\{15,3\} \times\{10,5,3\}$; (iv) $\{17,3\} \times\left\{8^{2}, 2,1^{2}\right\}$. The results are as follows: 
TABLE I: Inner products for $S_{4}$ irreps. The entries in each row give the coefficients of the inner products of the irreps listed in column 1 .

\begin{tabular}{|l|l|l|l|l|l|}
\cline { 2 - 6 } \multicolumn{1}{c|}{} & $\{4\}$ & $\{31\}$ & $\{22\}$ & $\left\{21^{2}\right\}$ & $\left\{1^{4}\right\}$ \\
\hline$\{4\}^{\prime}\{4\}^{\prime \prime}$ & 1 & & & & \\
$\{4\}^{\prime}\{31\}^{\prime \prime}$ & & 1 & & & \\
$\{4\}^{\prime}\left\{2^{2}\right\}^{\prime \prime}$ & & & 1 & & \\
$\{4\}^{\prime}\left\{21^{2}\right\}^{\prime \prime}$ & & & & 1 & \\
$\{4\}^{\prime}\left\{1^{4}\right\}^{\prime \prime}$ & & & & & 1 \\
\hline$\{31\}^{\prime}\{31\}^{\prime \prime}$ & 1 & 1 & 1 & 1 & \\
$\{31\}^{\prime}\left\{2^{2}\right\}^{\prime \prime}$ & & 1 & & 1 & \\
$\{31\}^{\prime}\left\{21^{2}\right\}^{\prime \prime}$ & & 1 & 1 & 1 & 1 \\
$\{31\}^{\prime}\left\{1^{4}\right\}^{\prime \prime}$ & & & & 1 & \\
\hline$\left\{2^{2}\right\}^{\prime}\left\{2^{2}\right\}^{\prime \prime}$ & 1 & & 1 & & 1 \\
$\left\{2^{2}\right\}^{\prime}\left\{21^{2}\right\}^{\prime \prime}$ & & 1 & & 1 & \\
$\left\{2^{2}\right\}^{\prime}\left\{1^{4}\right\}^{\prime \prime}$ & & & 1 & & \\
\hline$\left\{21^{2}\right\}^{\prime}\left\{21^{2}\right\}^{\prime \prime}$ & 1 & 1 & 1 & 1 & \\
$\left\{21^{2}\right\}^{\prime}\left\{1^{4}\right\}^{\prime \prime}$ & & 1 & & & \\
\hline$\left\{1^{4}\right\}^{\prime}\left\{1^{4}\right\}^{\prime \prime}$ & 1 & & & & \\
\hline
\end{tabular}

$$
\begin{aligned}
& \left\{5,4,2,1^{2}\right\} \times\left\{5,4,2,1^{2}\right\}=\{13\}+3\{12,1\}+10\{11,2\}+23\{10,3\}+41\{9,4\}+ \\
& 49\{8,5\}+35\{7,6\}+9\left\{11,1^{2}\right\}+45\{10,2,1\}+121\{9,3,1\}+83\left\{9,2^{2}\right\}+ \\
& 211\{8,4,1\}+276\{8,3,2\}+223\{7,5,1\}+461\{7,4,2\}+271\left\{7,3^{2}\right\}+99\left\{6^{2}, 1\right\}+ \\
& 385\{6,5,2\}+476\{6,4,3\}+250\left\{5^{2}, 3\right\}+188\left\{5,4^{2}\right\}+22\left\{10,1^{3}\right\}+123\left\{9,2,1^{2}\right\}+ \\
& 335\left\{8,3,1^{2}\right\}+288\left\{8,2^{2}, 1\right\}+523\left\{7,4,1^{2}\right\}+911\{7,3,2,1\}+305\left\{7,2^{3}\right\}+ \\
& 427\left\{6,5,1^{2}\right\}+1271\{6,4,2,1\}+845\left\{6,3^{2}, 1\right\}+891\left\{6,3,2^{2}\right\}+629\left\{5^{2}, 2,1\right\}+ \\
& 1094\{5,4,3,1\}+943\left\{5,4,2^{2}\right\}+846\left\{5,3^{2}, 2\right\}+248\left\{4^{3}, 1\right\}+623\left\{4^{2}, 3,2\right\}+ \\
& 249\left\{4,3^{3}\right\}+41\left\{9,1^{4}\right\}+234\left\{8,2,1^{3}\right\}+589\left\{7,3,1^{3}\right\}+556\left\{7,2^{2}, 1^{2}\right\}+ \\
& 762\left\{6,4,1^{3}\right\}+1527\left\{6,3,2,1^{2}\right\}+673\left\{6,2^{3}, 1\right\}+368\left\{5^{2}, 1^{3}\right\}+1573\left\{5,4,2,1^{2}\right\}+ \\
& 1175\left\{5,3^{2}, 1^{2}\right\}+1580\left\{5,3,2^{2}, 1\right\}+374\left\{5,2^{4}\right\}+842\left\{4^{2}, 3,1^{2}\right\}+942\left\{4^{2}, 2^{2}, 1\right\}+ \\
& 1097\left\{4,3^{2}, 2,1\right\}+632\left\{4,3,2^{3}\right\}+187\left\{3^{4}, 1\right\}+250\left\{3^{3}, 2^{2}\right\}+60\left\{8,1^{5}\right\}+ \\
& 322\left\{7,2,1^{4}\right\}+694\left\{6,3,1^{4}\right\}+698\left\{6,2^{2}, 1^{3}\right\}+663\left\{5,4,1^{4}\right\}+1521\left\{5,3,2,1^{3}\right\}+ \\
& 766\left\{5,2^{3}, 1^{2}\right\}+882\left\{4^{2}, 2,1^{3}\right\}+839\left\{4,3^{2}, 1^{3}\right\}+1266\left\{4,3,2^{2}, 1^{2}\right\}+426\left\{4,2^{4}, 1\right\}+ \\
& 472\left\{3^{3}, 2,1^{2}\right\}+380\left\{3^{2}, 2^{3}, 1\right\}+96\left\{3,2^{5}\right\}+68\left\{7,1^{6}\right\}+319\left\{6,2,1^{5}\right\}+ \\
& 546\left\{5,3,1^{5}\right\}+582\left\{5,2^{2}, 1^{4}\right\}+296\left\{4^{2}, 1^{5}\right\}+889\left\{4,3,2,1^{4}\right\}+511\left\{4,2^{3}, 1^{3}\right\}+ \\
& 262\left\{3^{3}, 1^{4}\right\}+444\left\{3^{2}, 2^{2}, 1^{3}\right\}+212\left\{3,2^{4}, 1^{2}\right\}+32\left\{2^{6}, 1\right\}+59\left\{6,1^{7}\right\}+ \\
& 224\left\{5,2,1^{6}\right\}+271\left\{4,3,1^{6}\right\}+314\left\{4,2^{2}, 1^{5}\right\}+254\left\{3^{2}, 2,1^{5}\right\}+191\left\{3,2^{3}, 1^{4}\right\}+ \\
& 42\left\{2^{5}, 1^{3}\right\}+38\left\{5,1^{8}\right\}+107\left\{4,2,1^{7}\right\}+70\left\{3^{2}, 1^{7}\right\}+101\left\{3,2^{2}, 1^{6}\right\}+ \\
& 31\left\{2^{4}, 1^{5}\right\}+17\left\{4,1^{9}\right\}+32\left\{3,2,1^{8}\right\}+15\left\{2^{3}, 1^{7}\right\}+5\left\{3,1^{10}\right\}+ \\
& 5\left\{2^{2}, 1^{9}\right\}+\left\{2,1^{11}\right\} .
\end{aligned}
$$




$$
\begin{aligned}
& \{11,5\} \times\{6,4,3,2,1\}=\{10,4,2\}+\left\{10,3^{2}\right\}+\{9,6,1\}+5\{9,5,2\}+ \\
& 7\{9,4,3\}+\{8,7,1\}+8\{8,6,2\}+17\{8,5,3\}+10\left\{8,4^{2}\right\}+4\left\{7^{2}, 2\right\}+ \\
& 16\{7,6,3\}+20\{7,5,4\}+11\left\{6^{2}, 4\right\}+8\left\{6,5^{2}\right\}+\left\{10,4,1^{2}\right\}+ \\
& 3\{10,3,2,1\}+\left\{10,2^{3}\right\}+5\left\{9,5,1^{2}\right\}+18\{9,4,2,1\}+13\left\{9,3^{2}, 1\right\}+ \\
& 13\left\{9,3,2^{2}\right\}+8\left\{8,6,1^{2}\right\}+38\{8,5,2,1\}+53\{8,4,3,1\}+43\left\{8,4,2^{2}\right\}+ \\
& 36\left\{8,3^{2}, 2\right\}+4\left\{7^{2}, 1^{2}\right\}+34\{7,6,2,1\}+79\{7,5,3,1\}+59\left\{7,5,2^{2}\right\}+ \\
& 49\left\{7,4^{2}, 1\right\}+103\{7,4,3,2\}+29\left\{7,3^{3}\right\}+39\left\{6^{2}, 3,1\right\}+28\left\{6^{2}, 2^{2}\right\}+ \\
& 62\{6,5,4,1\}+103\{6,5,3,2\}+72\left\{6,4^{2}, 2\right\}+64\left\{6,4,3^{2}\right\}+13\left\{5^{3}, 1\right\} \\
& 49\left\{5^{2}, 4,2\right\}+36\left\{5^{2}, 3^{2}\right\}+38\left\{5,4^{2}, 3\right\}+5\left\{4^{4}\right\}+\left\{10,3,1^{3}\right\} \\
& \left\{10,2^{2}, 1^{2}\right\}+8\left\{9,4,1^{3}\right\}+18\left\{9,3,2,1^{2}\right\}+7\left\{9,2^{3}, 1\right\}+18\left\{8,5,1^{3}\right\}+ \\
& 61\left\{8,4,2,1^{2}\right\}+43\left\{8,3^{2}, 1^{2}\right\}+53\left\{8,3,2^{2}, 1\right\}+10\left\{8,2^{4}\right\}+16\left\{7,6,1^{3}\right\}+ \\
& 84\left\{7,5,2,1^{2}\right\}+121\left\{7,4,3,1^{2}\right\}+121\left\{7,4,2^{2}, 1\right\}+105\left\{7,3^{2}, 2,1\right\}+ \\
& 50\left\{7,3,2^{3}\right\}+40\left\{6^{2}, 2,1^{2}\right\}+119\left\{6,5,3,1^{2}\right\}+111\left\{6,5,2^{2}, 1\right\}+ \\
& 79\left\{6,4^{2}, 1^{2}\right\}+215\{6,4,3,2,1\}+81\left\{6,4,2^{3}\right\}+67\left\{6,3^{3}, 1\right\}+77\left\{6,3^{2}, 2^{2}\right\}+ \\
& 53\left\{5^{2}, 4,1^{2}\right\}+116\left\{5^{2}, 3,2,1\right\}+41\left\{5^{2}, 2^{3}\right\}+101\left\{5,4^{2}, 2,1\right\}+99\left\{5,4,3^{2}, 1\right\}+ \\
& 106\left\{5,4,3,2^{2}\right\}+45\left\{5,3^{3}, 2\right\}+31\left\{4^{3}, 3,1\right\}+27\left\{4^{3}, 2^{2}\right\}+35\left\{4^{2}, 3^{2}, 2\right\}+ \\
& 8\left\{4,3^{4}\right\}+5\left\{9,3,1^{4}\right\}+5\left\{9,2^{2}, 1^{3}\right\}+18\left\{8,4,1^{4}\right\}+39\left\{8,3,2,1^{3}\right\} \\
& 17\left\{8,2^{3}, 1^{2}\right\}+25\left\{7,5,1^{4}\right\}+88\left\{7,4,2,1^{3}\right\}+63\left\{7,3^{2}, 1^{3}\right\}+84\left\{7,3,2^{2}, 1^{2}\right\}+ \\
& 21\left\{7,2^{4}, 1\right\}+12\left\{6^{2}, 1^{4}\right\}+80\left\{6,5,2,1^{3}\right\}+124\left\{6,4,3,1^{3}\right\}+134\left\{6,4,2^{2}, 1^{2}\right\}+ \\
& 121\left\{6,3^{2}, 2,1^{2}\right\}+74\left\{6,3,2^{3}, 1\right\}+10\left\{6,2^{5}\right\}+66\left\{5^{2}, 3,1^{3}\right\}+67\left\{5^{2}, 2^{2}, 1^{2}\right\}+ \\
& 53\left\{5,4^{2}, 1^{3}\right\}+162\left\{5,4,3,2,1^{2}\right\}+78\left\{5,4,2^{3}, 1\right\}+56\left\{5,3^{3}, 1^{2}\right\}+ \\
& 80\left\{5,3^{2}, 2^{2}, 1\right\}+24\left\{5,3,2^{4}\right\}+39\left\{4^{3}, 2,1^{2}\right\}+42\left\{4^{2}, 3^{2}, 1^{2}\right\}+56\left\{4^{2}, 3,2^{2}, 1\right\}+ \\
& 13\left\{4^{2}, 2^{4}\right\}+30\left\{4,3^{3}, 2,1\right\}+16\left\{4,3^{2}, 2^{3}\right\}+3\left\{3^{5}, 1\right\}+3\left\{3^{4}, 2^{2}\right\}+ \\
& \left\{9,2,1^{5}\right\}+8\left\{8,3,1^{5}\right\}+8\left\{8,2^{2}, 1^{4}\right\}+18\left\{7,4,1^{5}\right\}+39\left\{7,3,2,1^{4}\right\}+ \\
& 18\left\{7,2^{3}, 1^{3}\right\}+16\left\{6,5,1^{5}\right\}+61\left\{6,4,2,1^{4}\right\}+45\left\{6,3^{2}, 1^{4}\right\}+62\left\{6,3,2^{2}, 1^{3}\right\}+ \\
& 17\left\{6,2^{4}, 1^{2}\right\}+30\left\{5^{2}, 2,1^{4}\right\}+57\left\{5,4,3,1^{4}\right\}+64\left\{5,4,2^{2}, 1^{3}\right\}+61\left\{5,3^{2}, 2,1^{3}\right\}+ \\
& 40\left\{5,3,2^{3}, 1^{2}\right\}+7\left\{5,2^{5}, 1\right\}+12\left\{4^{3}, 1^{4}\right\}+40\left\{4^{2}, 3,2,1^{3}\right\}+21\left\{4^{2}, 2^{3}, 1^{2}\right\}+
\end{aligned}
$$$$
\begin{aligned}
& 17\left\{4,3^{3}, 1^{3}\right\}+25\left\{4,3^{2}, 2^{2}, 1^{2}\right\}+9\left\{4,3,2^{4}, 1\right\}+\left\{4,2^{6}\right\}+4\left\{3^{4}, 2,1^{2}\right\}+ \\
& 2\left\{3^{3}, 2^{3}, 1\right\}+\left\{8,2,1^{6}\right\}+5\left\{7,3,1^{6}\right\}+5\left\{7,2^{2}, 1^{5}\right\}+8\left\{6,4,1^{6}\right\}+18\left\{6,3,2,1^{5}\right\}+ \\
& 8\left\{6,2^{3}, 1^{4}\right\}+4\left\{5^{2}, 1^{6}\right\}+18\left\{5,4,2,1^{5}\right\}+14\left\{5,3^{2}, 1^{5}\right\}+19\left\{5,3,2^{2}, 1^{4}\right\}+ \\
& 5\left\{5,2^{4}, 1^{3}\right\}+8\left\{4^{2}, 3,1^{5}\right\}+9\left\{4^{2}, 2^{2}, 1^{4}\right\}+10\left\{4,3^{2}, 2,1^{4}\right\}+6\left\{4,3,2^{3}, 1^{3}\right\}+ \\
& \left\{4,2^{5}, 1^{2}\right\}+\left\{3^{4}, 1^{4}\right\}+\left\{3^{3}, 2^{2}, 1^{3}\right\}+\left\{6,3,1^{7}\right\}+\left\{6,2^{2}, 1^{6}\right\}+ \\
& \left\{5,4,1^{7}\right\}+3\left\{5,3,2,1^{6}\right\}+\left\{5,2^{3}, 1^{5}\right\}+\left\{4^{2}, 2,1^{6}\right\}+\left\{4,3^{2}, 1^{6}\right\}+\left\{4,3,2^{2}, 1^{5}\right\} .
\end{aligned}
$$ 


$$
\begin{aligned}
& \{15,3\} \times\{10,5,3\}=\{13,5\}+\{12,6\}+\{11,7\}+\{10,8\}+\{13,4,1\}+ \\
& \{13,3,2\}+3\{12,5,1\}+4\{12,4,2\}+2\left\{12,3^{2}\right\}+4\{11,6,1\}+8\{11,5,2\}+ \\
& 7\{11,4,3\}+3\{10,7,1\}+8\{10,6,2\}+11\{10,5,3\}+5\left\{10,4^{2}\right\}+\{9,8,1\}+ \\
& 4\{9,7,2\}+8\{9,6,3\}+7\{9,5,4\}+\left\{8^{2}, 2\right\}+3\{8,7,3\}+4\{8,6,4\}+ \\
& 2\left\{8,5^{2}\right\}+\left\{7^{2}, 4\right\}+\{7,6,5\}+2\left\{12,4,1^{2}\right\}+2\{12,3,2,1\}+4\left\{11,5,1^{2}\right\}+ \\
& 7\{11,4,2,1\}+4\left\{11,3^{2}, 1\right\}+2\left\{11,3,2^{2}\right\}+4\left\{10,6,1^{2}\right\}+11\{10,5,2,1\}+ \\
& 11\{10,4,3,1\}+5\left\{10,4,2^{2}\right\}+3\left\{10,3^{2}, 2\right\}+2\left\{9,7,1^{2}\right\}+7\{9,6,2,1\}+ \\
& 12\{9,5,3,1\}+5\left\{9,5,2^{2}\right\}+5\left\{9,4^{2}, 1\right\}+6\{9,4,3,2\}+\left\{9,3^{3}\right\}+2\{8,7,2,1\}+ \\
& 5\{8,6,3,1\}+2\left\{8,6,2^{2}\right\}+5\{8,5,4,1\}+4\{8,5,3,2\}+2\left\{8,4^{2}, 2\right\}+\left\{8,4,3^{2}\right\}+ \\
& \left\{7^{2}, 3,1\right\}+\{7,6,4,1\}+\{7,6,3,2\}+\left\{7,5^{2}, 1\right\}+\{7,5,4,2\}+\left\{7,5,3^{2}\right\}+ \\
& \left\{11,4,1^{3}\right\}+\left\{11,3,2,1^{2}\right\}+2\left\{10,5,1^{3}\right\}+4\left\{10,4,2,1^{2}\right\}+2\left\{10,3^{2}, 1^{2}\right\}+ \\
& \left\{10,3,2^{2}, 1\right\}+\left\{9,6,1^{3}\right\}+4\left\{9,5,2,1^{2}\right\}+4\left\{9,4,3,1^{2}\right\}+2\left\{9,4,2^{2}, 1\right\}+ \\
& \left\{9,3^{2}, 2,1\right\}+\left\{8,6,2,1^{2}\right\}+2\left\{8,5,3,1^{2}\right\}+\left\{8,5,2^{2}, 1\right\}+\left\{8,4^{2}, 1^{2}\right\}+ \\
& \{8,4,3,2,1\}+\left\{9,4,2,1^{3}\right\} .
\end{aligned}
$$

$$
\begin{aligned}
& \{17,3\} \times\left\{8^{2}, 2,1^{2}\right\}=\{10,9,1\}+2\{10,8,2\}+\{10,7,3\}+2\left\{9^{2}, 2\right\}+ \\
& 3\{9,8,3\}+\{9,7,4\}+\left\{8^{2}, 4\right\}+\left\{11,7,1^{2}\right\}+\{11,6,2,1\}+3\left\{10,8,1^{2}\right\}+ \\
& 5\{10,7,2,1\}+2\{10,6,3,1\}+2\left\{10,6,2^{2}\right\}+3\left\{9^{2}, 1^{2}\right\}+10\{9,8,2,1\}+ \\
& 8\{9,7,3,1\}+6\left\{9,7,2^{2}\right\}+2\{9,6,4,1\}+3\{9,6,3,2\}+7\left\{8^{2}, 3,1\right\}+6\left\{8^{2}, 2^{2}\right\}+ \\
& 4\{8,7,4,1\}+7\{8,7,3,2\}+\{8,6,5,1\}+2\{8,6,4,2\}+\left\{8,6,3^{2}\right\}+\left\{7^{2}, 4,2\right\}+ \\
& \left\{7^{2}, 3^{2}\right\}+\left\{11,6,1^{3}\right\}+\left\{11,5,2,1^{2}\right\}+4\left\{10,7,1^{3}\right\}+4\left\{10,6,2,1^{2}\right\}+ \\
& \left\{10,5,3,1^{2}\right\}+\left\{10,5,2^{2}, 1\right\}+7\left\{9,8,1^{3}\right\}+13\left\{9,7,2,1^{2}\right\}+6\left\{9,6,3,1^{2}\right\}+ \\
& 5\left\{9,6,2^{2}, 1\right\}+\left\{9,5,4,1^{2}\right\}+\{9,5,3,2,1\}+11\left\{8^{2}, 2,1^{2}\right\}+12\left\{8,7,3,1^{2}\right\}+ \\
& 11\left\{8,7,2^{2}, 1\right\}+4\left\{8,6,4,1^{2}\right\}+6\{8,6,3,2,1\}+2\left\{8,6,2^{3}\right\}+\left\{8,5^{2}, 1^{2}\right\}+ \\
& \{8,5,4,2,1\}+2\left\{7^{2}, 4,1^{2}\right\}+5\left\{7^{2}, 3,2,1\right\}+2\left\{7^{2}, 2^{3}\right\}+\{7,6,4,2,1\}+ \\
& 5\left\{9,6,2,1^{3}\right\}+\left\{9,5,3,1^{3}\right\}+\left\{9,5,2^{2}, 1^{2}\right\}+5\left\{8^{2}, 1^{4}\right\}+11\left\{8,7,2,1^{3}\right\}+ \\
& 5\left\{8,6,3,1^{3}\right\}+4\left\{8,6,2^{2}, 1^{2}\right\}+\left\{8,5,4,1^{3}\right\}+\left\{8,5,3,2,1^{2}\right\}+4\left\{7^{2}, 3,1^{3}\right\}+ \\
& 5\left\{7^{2}, 2^{2}, 1^{2}\right\}+\left\{7,6,4,1^{3}\right\}+2\left\{7,6,3,2,1^{2}\right\}+\left\{7,6,2^{3}, 1\right\}+\left\{9,6,1^{5}\right\}+ \\
& 3\left\{8,7,1^{5}\right\}+2\left\{8,6,2,1^{4}\right\}+3\left\{7^{2}, 2,1^{4}\right\}+\left\{7,6,3,1^{4}\right\}+\left\{7,6,2^{2}, 1^{3}\right\}+ \\
& \left\{7^{2}, 1^{6}\right\} .
\end{aligned}
$$

In addition, Table 2 gives the number of terms and the highest multiplicities for some selected inner products with $n \leq 20$. As seen from Eqs. (24)-(27) and Table 2, for $n>10$ the number of terms in the inner products start becoming large and also the multiplicities big. For example, as seen from Eq. (24), for $\{11,5\} \times\{6,4,3,2,1\}$ there are 164 terms and the multiplicities for the Schur functions $\{6,4,3,2,1\},\left\{6,5,2^{2}, 1\right\},\{7,5,3,1\},\left\{5,4,2^{3}, 1\right\}$ and $\left\{4^{2}, 3,2,1^{3}\right\}$ are $215,111,79,78$ and 40 respectively. Similarly in $\left\{5,4,2,1^{2}\right\} \times\left\{5,4,2,1^{2}\right\}$ there are 100 terms and highest multiplicity is 1580 . Let us mention that full tables for $n \leq 20$ are available from download in the homepage of one of the authors (www.ift.unesp.br/users/jaca)

\section{CONCLUSIONS}

In this paper discussed are two methods (called I and II in Sections II and III respectively) for calculating inner products of Schur functions in terms of outer products and 
TABLE II: Highest multiplicities for selected inner products. Listed in column 2 are the number of terms in the inner product, in column 3 the highest multiplicity and in column 4 the term with this multiplicity.

\begin{tabular}{|c|c|c|c|}
\hline inner product & no. terms & highest mult. & term with highest mult. \\
\hline$\{4,3,2,1\} \times\{4,3,2,1\}$ & 42 & 117 & $\{4,3,2,1\}$ \\
$\{5,3,2,1\} \times\{5,3,2,1\}$ & 55 & 312 & $\{5,3,2,1\}$ \\
$\left\{5,3,2,1^{2}\right\} \times\left\{5,3,2,1^{2}\right\}$ & 77 & 945 & $\left\{5,3,2,1^{2}\right\}$ \\
$\left\{6,4,2,1^{2}\right\} \times\left\{6,4,2,1^{2}\right\}$ & 133 & 3985 & $\left\{6,4,2,1^{2}\right\}$ \\
$\{5,4,3,2,1\} \times\{5,4,3,2,1\}$ & 176 & 18269 & $\{5,4,3,2,1\}$ \\
$\{6,4,3,2,1\} \times\{6,4,3,2,1\}$ & 239 & 72973 & $\{6,4,3,2,1\}$ \\
$\left\{6,4,3,2,1^{2}\right\} \times\left\{6,4,3,2,1^{2}\right\}$ & 297 & 324133 & $\left\{6,4,3,2,1^{2}\right\}$ \\
$\left\{7,4,3,2,1^{2}\right\} \times\left\{7,4,3,2,1^{2}\right\}$ & 384 & 684784 & $\left\{7,4,3,2,1^{2}\right\}$ \\
$\left\{7,5,3,2,1^{2}\right\} \times\left\{7,5,3,2,1^{2}\right\}$ & 488 & 2274958 & $\left\{7,5,3,2,1^{2}\right\}$ \\
$\left\{8,6,4,1^{2}\right\} \times\left\{7,5,3,2^{2}, 1\right\}$ & 613 & 17055035 & $\left\{6,5,4,2,1^{3}\right\}$ \\
\hline
\end{tabular}

plethysms. One of these (II) is derived from a recent analysis of the $S O(8)$ proton-neutron pairing model of atomic nuclei [1]. It is useful to mention that in general method I is preferable to method II because it needs only outer products (hence more efficient computationally) while method II needs plethysms. However we could use method II as we have a great bank of plethysms [16]. Also method II, as discussed in [1], can give in many situations analytical results. Similarly method I can be used to obtain analytical results for inner products involving $S_{n}$ irreps with small number of parts as for example those given in Eqs. (7-167)-(7-170) in [3]. We have generated tabulations for inner products of Schur functions of degree $n \leq 20$ and this represents a major advance since the tabulations by James and Kerber [22] and Wybourne [5] for $n \leq 9$. Thus, with our previous work $[10,11,14]$ we have generated tabulations for plethysms [16] and now with the present paper we have tabulations for inner products. Finally let us mention that the numerical results for large $n$, giving large multiplicities (see Table 2), can be useful for the following: (i) to test formulas derived for simple class of irreps (for example references [3],[17]); (ii) to examine the systematics of the multiplicities, as to see if it is possible to derive formulas for some other class of irreps; (iii) to examine the possibility of identifying some statistical structure for the multiplicities. They are also useful for developing and testing statistical models for these and this belongs to the emerging topic of statistical group theory. See [19-21] for some papers on this subject.

For large $N$ the tables are very long and therefore unpractical for hand calculations. Their use is for computer calculation, when the data are easily obtained from the files stored in the computer memory. For this reason they are available, in computer form, for download in the already mentioned web address, together with programs for handling them.
[1] V.K.B. Kota and J.A. Castilho Alcarás, Nucl. Phys. A764, 181 (2006).

[2] V.K.B. Kota, in Focus on Boson Research, edited by A.V. Ling (Nova Science Publishers Inc., New York, 2006), p. 57.

[3] M. Hamermesh, Group Theory and its Application to Physical Problems (Addison-Wesley, Massachusetts, 1962).

[4] D.E. Littlewood, J. London Math. Soc. 31, 89 (1956).

[5] B.G. Wybourne, Symmetry Principles and Atomic Spectroscopy (Wiley, New York, 1970).

[6] M.J. Carvalho and S.D. Agostino, J. Phys. A: Math. Gen. 34, 1375 (2001).

[7] D. E. Littlewood, The Theory of Group Characters and Matrix Representations of Groups, 2nd edition (Oxford University Press, Oxford, 1950); reprinted by AMS Chelsea publishing (American Mathematical Society, Providence, Rhode Island, 2006).

[8] I. G. MacDonald, Symmetry Functions and Hall Polynomials, 2nd edition (Clarendon, Oxford, 1995).

[9] D.E. Littlewood, J. London Math. Soc. 11, 49 (1936).

[10] J.A. Castilho Alcarás, J. Tambergs J, T. Krasta, J. Ruža J and O. Katkevičius, Braz. J. Phys. 32, 641 (2002).

[11] J.A. Castilho Alcarás, J. Tambergs J, T. Krasta, J. Ruža J and O. Katkevičius, J. Math. Phys. 44, 5296 (2003).

[12] S.M. Christensen and Associates Inc., Schur Group The- ory Software by B.G. Wybourne (E-mail: steve@smc.vnet.in; http://smc.vnet.net/Schur.html).

[13] M.J. Carvalho and S.D. Agostino, Comp. Phys. Comm. 141, 282 (2001).

[14] J.A. Castilho Alcarás, J. Tambergs J, T. Krasta, J. Ruža J and O. Katkevičius, J. Phys. A: Math. Gen. 38, 7501 (2005).

[15] D.E. Littlewood and A.R. Richardson, Phil. Trans. R. Soc. A 233, 99 (1934).

[16] J.A. Castilho Alcarás, Bank of Plethysms (available on request).

[17] M.H. Rosas, J. Algebraic Combinatorics 14, 153 (2001).

[18] D.E. Littlewood, J. London Math. Soc. 32, 18 (1957).

[19] P. Erdos and P. Turan, Periodica Mathematica Hungarica 2, 149 (1972).

[20] J.G. Cleary and B.G. Wybourne, J. Math. Phys. 12, 45 (1971); M.G. Hirst and B.G. Wybourne 1986 J. Phys. A 19, 1545 (1986).

[21] V.K.B. Kota, K.B.K. Mayya and J.A. Castilho Alcarás J. Phys. A: Math. Theor. 42, 145201 (2009).

[22] G. James and A. Kerber, The representation theory of the symmetric group(Encyclopedia of Mathematics Series, vol 16, 1981) (Reading, MA: Addison-Wesley). 